\title{
Validation of the JCOG prognostic index in advanced gastric cancer using individual patient data from the SPIRITS and G-SOX trials
}

\author{
Daisuke Takahari $^{1}$ (D) Junki Mizusawa ${ }^{2} \cdot$ Wasaburo Koizumi $^{3} \cdot$ Ichinosuke Hyodo $^{4}$ • \\ Narikazu Boku ${ }^{5}$
}

Received: 25 December 2016/Accepted: 5 February 2017/Published online: 16 February 2017

(c) The International Gastric Cancer Association and The Japanese Gastric Cancer Association 2017

\begin{abstract}
Background In a phase III study for advanced gastric cancer (AGC), the Japan Clinical Oncology Group (JCOG) 9912 trial, we previously identified the following four prognostic factors-performance status $\geq 1$, number of metastatic sites $\geq 2$, no prior gastrectomy, and abnormal serum alkaline phosphatase levels-and proposed a prognostic index (good risk with 0 or 1 factor, moderate risk with 2 or 3 factors, and poor risk with all 4 factors). To assess the generalizability of this index, we attempted an external validation study using an independent data set. Methods Individual patient data from the SPIRITS and G-SOX trials were applied to the JCOG prognostic index. The accuracy of the index for predicting survival was assessed by the Cox proportional hazards model.

Results The available data were obtained from 936 (94.5\%) of the 990 patients in these trials. The three risk groups categorized by the JCOG prognostic index
\end{abstract}

Presented in part at the 2016 Annual Meeting of the American Society of Clinical Oncology, 3-7 June 2016, Chicago, IL, USA.

Daisuke Takahari

daisuke.takahari@jfcr.or.jp

1 Department of Gastroenterology, Cancer Institute Hospital of Japanese Foundation for Cancer Research, 3-8-31 Ariake, Koto ward, Tokyo 135-8550, Japan

2 Biostatistics Division, Center for Research Administration and Support, National Cancer Center, Tokyo, Japan

3 Department of Gastroenterology, Kitasato University School of Medicine, Sagamihara, Japan

4 Division of Gastroenterology, University of Tsukuba, Tsukuba, Japan

5 Division of Gastrointestinal Medical Oncology, National Cancer Center Hospital, Tokyo, Japan demonstrated highly significant survival differences; the hazard ratios $(95 \%$ confidence interval) were 1.71 $(1.46-2.01)$ between the good $(n=338)$ and moderate $(n=537)$ risk groups and $3.32(2.47-4.46)$ between good and poor $(n=61)$ risk groups. The median overall survival times of the good, moderate, and poor risk groups were 17.2, 12.0, and 7.8 months, respectively.

Conclusions The JCOG prognostic index was externally validated and can be widely utilized for clinical trials. Further studies are needed to apply this index to the Western population.

Keywords Prognostic index - Prognostic factor · Advanced gastric cancer - Chemotherapy

\section{Introduction}

Despite a steady decline in the incidence and mortality rates of gastric cancer (GC), it remains a major health problem, causing 951,600 new cases and 723,100 deaths worldwide in 2012 [1], with 132,000 new cases and 50,000 deaths occurring in Japan [2] in 2012. For patients with advanced gastric cancer (AGC), the primary treatment strategy is systemic chemotherapy, and fluoropyrimidine plus platinum \pm trastuzumab has been regarded to be the standard first-line chemotherapy worldwide [3-5]. However, the outcome of these patients remains unsatisfactory; therefore, more effective regimens are needed, with assessment by clinical trials.

Prognostic indices are not only useful for estimating the prognosis of each patient, but are also applicable to selecting the optimal treatment strategy and stratifying patients with several types of cancer for clinical trials [6-8]. 
A prognostic index for AGC that was based on clinical trials conducted in the 1990s was proposed by the Royal Marsden Hospital (RMH) in 2004 [9]; the index consists of the following four independent risk factors for survival: Eastern Cooperative Oncology Group (ECOG) performance status (PS) $\geq 2$, liver metastasis, peritoneal metastasis, and serum alkaline phosphatase (ALP) $\geq 100 \mathrm{U} / \mathrm{l}$. Patients were classified into the following three groups by the number of risk factors: low risk (no risk factors), moderate risk ( 1 or 2 risk factors), and high risk ( 3 or 4 risk factors), which subsequently showed significant survival differences across the groups. However, the limitations of this index are that it was developed using data from Western patients only, and $30 \%$ of the patients had esophageal cancer.

Japan Clinical Oncology Group (JCOG) 9912 was a large randomized trial for patients with metastatic or recurrent gastric cancer that investigated the superiority of irinotecan plus cisplatin (IP) and the noninferiority of oral S-1 compared with continuous infusion of 5-fluorouracil (5-FUci) for patients with metastatic or recurrent gastric cancer [10]. This trial demonstrated that S-1 was not inferior to 5-FUci [hazard ratio (HR): 0.83 (95\% confidence interval, CI, 0.68-1.01); $P=0.0005$ for noninferiority] in terms of overall survival (OS), but IP was also not superior [HR: 0.85 (95\% CI $0.70-1.04$ ); $P=0.0552$ for superiority].

Ten years after the introduction of the RMH index, a few articles appeared that reported on prognostic factors [11-13]; however, none of these were based on data from patients participating in randomized controlled trials (RCTs). We used the data from 760 patients enrolled in JCOG9912 to identify the following four risk factors: PS $\geq 1$, number of metastatic sites $\geq 2$, no prior gastrectomy, and serum ALP level $>$ normal range. We also proposed a 'JCOG prognostic index' that was based on these factors, as follows: good risk ( $0-1$ factor), moderate risk (2-3 factors), and poor risk (4 factors) [14]. The OS curves of the three risk groups based on the number of risk factors showed significantly good separation in the JCOG9912 trial. However, this prognostic index should be validated by other cohorts.

The aim of this study was to validate the JCOG prognostic index in a combined patient cohort from two other Japanese phase III trials for AGC, the "S-1 Plus cisplatin versus S-1 In RCT In the Treatment for Stomach cancer" (SPIRITS) trial [15] and the "The Randomized Phase III Study Comparing Oxaliplatin plus S-1 with Cisplatin plus $\mathrm{S}-1$ in Chemotherapy-naïve Patients with Advanced Gastric Cancer" (G-SOX) trial [16]. We additionally evaluated the RMH index in this study.

\section{Methods}

\section{Patients}

The SPIRITS trial was a phase III trial that compared S-1 with S-1 + cisplatin (SP) [HR: 0.77 (95\% CI 0.61-0.98); $P=0.04$ ] [15], and the G-SOX trial compared SP with S-1 + oxaliplatin (SOX) [HR: 0.96 (95\% CI 0.80-1.14)] [16].

We used the individual data sets from these two Japanese phase III studies to validate the JCOG prognostic index. The details of the inclusion/exclusion criteria and treatments for patients enrolled in the SPIRITS and the G-SOX trials were published previously $[15,16]$. Eligible patients were enrolled between 2002 and 2004 in the SPIRITS and between 2010 and 2011 in the G-SOX trial. The patients analyzed in this study were those having complete data on the four independent poor prognostic factors in the JCOG index (PS, number of metastatic sites, prior gastrectomy, and ALP level) and RMH index (PS, liver metastasis, peritoneal metastasis, and ALP level). Metastatic sites were reported by each investigator according to the Response Evaluation Criteria in Solid Tumors (RECIST), version 1.0, with specification of all target and non-target lesions on the case report form of each enrolled patient. The investigator prospectively recorded the presence or absence of such metastatic sites as the cervical, mediastinal, abdominal, and superficial lymph nodes; lung; liver; peritoneum; ovary; adrenal gland; bone; skin; and others. To compute the total number of metastatic sites in each patient, each organ was counted separately, and all lymph node metastases were counted as a single site, regardless of the location.

\section{Statistical analysis}

OS was measured from the date of randomization to the date of death from any cause and censored at the date of last contact for a surviving patient. OS was estimated by the Kaplan-Meier method. Survival curves covered up to 36 months. To investigate the impact of each factor on OS in the JCOG and RMH prognostic indices, the HR of each of the four prognostic factors stratified by regimens was calculated by the multivariable Cox proportional hazards model. To investigate the applicability of the JCOG and $\mathrm{RMH}$ prognostic indices, stratified Cox proportional hazards models, with trials and regimens as strata, were conducted for all patients and separately in each study. All $P$ values were two-sided and were considered statistically significant at less than 5\%. Any data that could identify a patient were deleted from the databases. This analysis was 
approved by the multicenter Research and Ethics Committees of the Cancer Institute Hospital.

\section{Results}

This study included 936 patients (S-1 arm, $n=150$; SP arm, $n=470$; SOX arm, $n=316$ ), whose complete individual data were available for multivariate analyses and accounted for $94.5 \%$ of the total of 990 patients randomized in these trials (Fig. 1). Table 1 shows the baseline characteristics of the participants. Of 936 patients, 660 $(71 \%)$ showed a PS of $0,394(42 \%)$ had 0 or 1 metastatic sites, $285(30 \%)$ had prior gastrectomy, and $288(31 \%)$ had an ALP level lower than the upper limit of the normal range. The distributions of risk groups by the JCOG index (good/moderate/poor) were 338/537/61, respectively, and by the RMH index (good/moderate/poor) were 314/598/24, respectively. Of 936 patients, $774(82.7 \%)$ died by the cutoff date for final data. The median survival time (MST) for all analyzed patients was 13.2 months (95\% CI 12.3-14.1 months) (Fig. 2).

Table 2(A), (B) shows the impact on survival of each prognostic factor in this combined multivariate analysis. All four risk factors in the JCOG index, including ALP level higher than the normal range, remained highly significant prognostic factors in this data set (Table 2A). On the other hand, peritoneal metastasis and ALP $\geq 100 \mathrm{U} / 1$ in the $\mathrm{RMH}$ index did not maintain significance (Table $2 \mathrm{~B}$ ).

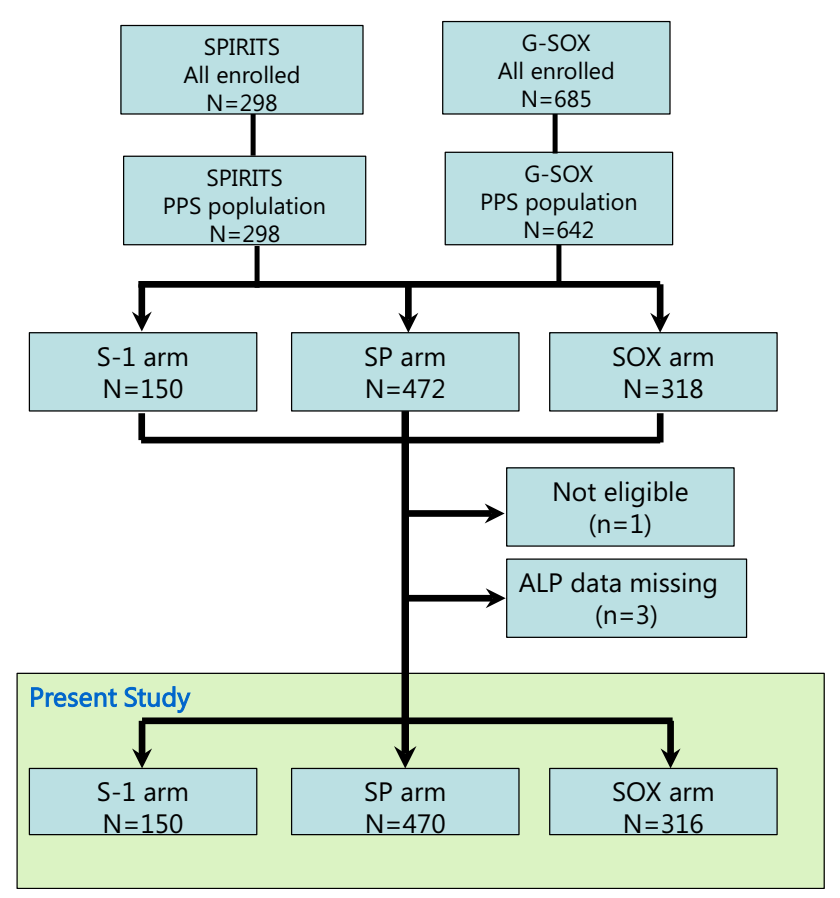

Fig. 1 CONSORT diagram
The three JCOG prognostic index risk groups clearly showed separate survival curves associated with an MST of 17.2 (95\% CI 15.2-18.4), 12.0 (95\% CI 11.3-13.2), and 7.8 (95\% CI 6.0-8.8) months, respectively (Fig. 3a). The Cox regression analysis found $\mathrm{HRs}(95 \% \mathrm{CI})$ of the moderate and poor risk groups were $1.71(1.46-2.01)(P<0.001$, $n=5370)$ and $3.32(2.47-4.46) \quad(P<0.001, n=61)$, respectively, compared with the good risk group $(n=338)$ (Table 3A). The RMH prognostic index showed that the poor risk group showed significantly shorter survival than the good risk group; the good versus moderate risk group did not show significant survival differences [compared with the good risk group ( $n=14)$; HR $(95 \% \mathrm{CI}) ; 1.10$ (0.94-1.28), $P=0.23$ for the moderate risk $(n=598)$ and 1.98 (1.27-3.10), $P=0.003$ for the poor risk $(n=24)]$, associated with an MST of 14.9 (95\% CI 12.9-16.3), 13.0 (95\% CI 11.9-13.9) and 6.9 (95\% CI 3.6-11.4) months, respectively (Fig. 3b; Table 3B).

In an analysis restricted to patients of the SPIRITS trial, the JCOG prognostic index identified highly significant survival differences among the three risk groups [compared with the good risk group ( $n=160)$; HR, $(95 \% \mathrm{CI}) ; 1.65$ (1.29-2.13), $P<0.001$ for the moderate risk group ( $n=125)$; and 4.77 (2.66-8.57), $P<0.001$ for the poor risk group $(n=13)]$, associated with an MST of $13.7(95 \% \mathrm{CI}$ 11.7-16.1), 11.2 (95\% CI 9.6-13.3), and 5.3 (95\% CI 2.2-8.7) months, respectively. An analysis of patients in the G-SOX trial similarly identified highly significant survival differences [compared with the good risk group $(n=178)$; HR, (95\% CI); 1.76 (1.44-2.19), $P<0.001$ for the moderate risk group $(n=412)$; and 3.11 (2.20-4.41), $P<0.001$ for the poor risk group $(n=48)]$, associated with an MST of 18.4 (95\% CI 17.2-22.3), 12.4 (95\% CI 11.4-13.8), and 8.0 (95\% CI 6.6-10.2) months, respectively.

Similar to the combined analysis, good and moderate risk groups categorized by the $\mathrm{RMH}$ prognostic index showed no significant survival differences except comparison between the good and poor risk group in the SPIRITS trial. As follows, in the SPIRITS trial, compared with the good risk group $(n=88)$; HR, (95\% CI); 1.07 (0.82-1.39), $P=0.62$ for the moderate risk group ( $n=204)$; and 5.60 (2.41-13.02), $P<0.001$ for the poor risk group $(n=6)]$, associated with an MST of $12.2(95 \%$ CI 9.6-15.8), 12.3 (95\% CI 10.5-14.0), and 4.3 (95\% CI 0.4-11.2) months, respectively. In the G-SOX trial, compared with the good risk group $(n=226)$; HR, $(95 \% \mathrm{CI})$; $1.11(0.92-1.34), P=0.27$ for the moderate risk group; ( $n=394)$ and $1.53(0.90-2.59), P=0.12$ for the poor risk group $(n=18)]$; associated with an MST of $15.3(95 \% \mathrm{CI}$ 13.3-17.4), 13.2 (95\% CI 11.9-14.4), and 8.7(95\% CI 5.7-12.5) months, respectively. 
Table 1 Patient characteristics

\begin{tabular}{|c|c|c|c|c|}
\hline $\begin{array}{l}\text { Chemotherapy regimen } \\
\text { Clinical trials }\end{array}$ & $\begin{array}{l}\text { S-1 } \\
\text { SPIRITS }\end{array}$ & $\begin{array}{l}\text { S-1 + cisplatin } \\
\text { SPIRITS } \\
\text { G-SOX }\end{array}$ & $\begin{array}{l}\text { S-1 + oxaliplatin } \\
\text { G-SOX }\end{array}$ & Total \\
\hline No. of patients & 150 & 470 & 316 & 936 \\
\hline \multicolumn{5}{|l|}{ Gender } \\
\hline Male & 116 & 344 & 238 & 698 \\
\hline Female & 34 & 126 & 78 & 238 \\
\hline \multicolumn{5}{|l|}{ ECOG PS } \\
\hline 0 & 106 & 332 & 222 & 660 \\
\hline 1 & 39 & 130 & 91 & 260 \\
\hline 2 & 5 & 8 & 3 & 16 \\
\hline \multicolumn{5}{|l|}{ Liver metastasis } \\
\hline No & 114 & 290 & 193 & 597 \\
\hline Yes & 36 & 180 & 123 & 339 \\
\hline \multicolumn{5}{|l|}{ Peritoneal metastasis } \\
\hline No & 95 & 353 & 255 & 703 \\
\hline Yes & 55 & 117 & 61 & 233 \\
\hline \multicolumn{5}{|l|}{ No. of metastatic sites } \\
\hline 0,1 & 96 & 197 & 101 & 394 \\
\hline$>2$ & 54 & 273 & 215 & 542 \\
\hline \multicolumn{5}{|l|}{ Gastrectomy } \\
\hline No & 77 & 331 & 243 & 651 \\
\hline Yes & 73 & 139 & 73 & 285 \\
\hline \multicolumn{5}{|l|}{ ALP } \\
\hline$<\mathrm{ULN}$ & 105 & 327 & 216 & 658 \\
\hline$\geq \mathrm{ULN}$ & 45 & 143 & 100 & 288 \\
\hline \multicolumn{5}{|l|}{ JCOG index } \\
\hline 0,1 & 78 & 172 & 88 & 338 \\
\hline 2,3 & 67 & 264 & 206 & 537 \\
\hline 4 & 5 & 34 & 22 & 61 \\
\hline \multicolumn{5}{|l|}{ RMH index } \\
\hline 0 & 48 & 151 & 115 & 314 \\
\hline 1,2 & 101 & 305 & 192 & 598 \\
\hline 3,4 & 1 & 14 & 9 & 24 \\
\hline
\end{tabular}

ECOG PS Eastern Cooperative Oncology Group performance status, ALP alkaline phosphatase, JCOG Japan Clinical Oncology Group, $U L N$ upper limit of normal

\section{Discussion}

Our current analysis, which was based on combined data from two prospective randomized controlled trials, proved that all four risk factors (PS $\geq 1$, metastatic sites $\geq 2$, no prior gastrectomy, and elevated ALP level) remained highly significant and validated the JCOG prognostic index. The OS curves of patients stratified into three risk groups (low, 0-1 risk factors; moderate, $2-3$ risk factors; high, 4 risk factors) showed significant separation, whereas the RMH prognostic index did not show significant separation between the OS of patients stratified into the good and moderate risk groups. Moreover, this trend was also preserved in the analyses of patients in each trial. The
JCOG9912 trial based on which the JCOG index was constructed was a phase III study of the previous era and did not contain the modern S-1/platinum combinations. Nevertheless, the JCOG index served well as a prognostic indicator among patients who were treated by the state-ofthe-art combination regimens that are currently used as first-line treatments in Japan and Asia.

There are several reasons why the JCOG index was validated by the two trials, whereas the RMH index was not validated. First, most of the patients participating in Japanese clinical trials had PS $\leq 1$. Indeed, only $16(2 \%)$ of 936 patients had PS of 2 in the validation set, whereas the data set establishing the RMH index included $23 \%$ of patients with PS of 2. In recent phase III trials of gastric cancer, the 


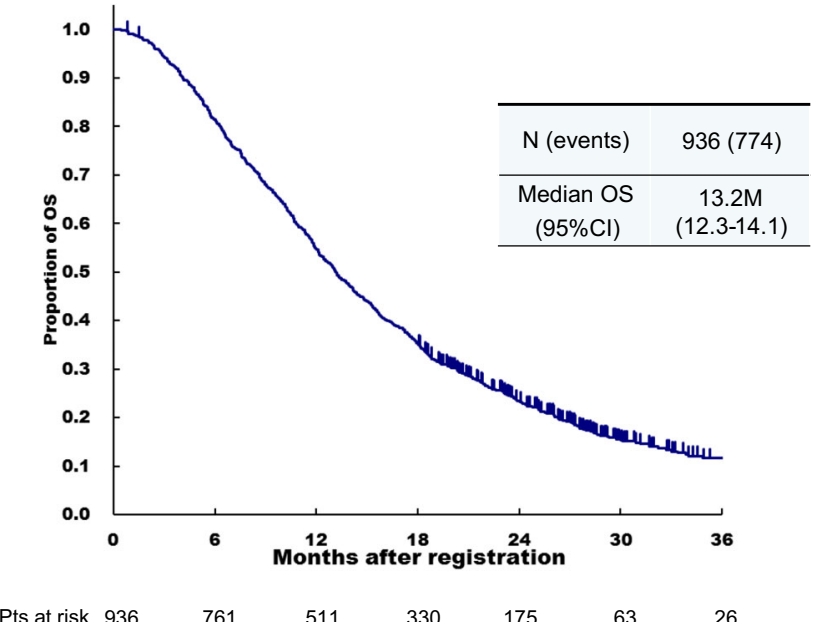

Fig. 2 Survival curve of the 936 patients with complete data for baseline factors and laboratory tests for the multivariate analysis. $O S$ overall survival, $C I$ confidence interval

Table 2 Multivariate analysis of survival

\begin{tabular}{lccc}
\hline Factor & HR & $95 \%$ CI & $P$ \\
\hline (A) & & & \\
PS 1, 2 (vs. 0) & 1.44 & $1.23-1.67$ & $<0.0001$ \\
No. of metastatic sites $\geq 2$ (vs. 0, 1) & 1.45 & $1.24-1.71$ & $<0.0001$ \\
Prior gastrectomy (-) (vs. (+)) & 1.55 & $1.31-1.84$ & $<0.0001$ \\
ALP $\geq$ ULN (vs. $<$ ULN) & 1.19 & $1.02-1.38$ & 0.03 \\
(B) & & & \\
PS 2 (vs. 0, 1) & 2.09 & $1.25-3.50$ & 0.0051 \\
Liver metastasis yes (vs. no) & 1.34 & $1.24-1.71$ & 0.0002 \\
Peritoneal metastasis yes (vs. no) & 1.01 & $0.85-1.21$ & 0.89 \\
ALP $\geq$ ULN (vs. < ULN) & 1.14 & $0.98-1.33$ & 0.09 \\
\hline
\end{tabular}

(A) JCOG index, (B) RMH index

$P S$ Eastern Cooperative Oncology Group performance status, $A L P$ alkaline phosphatase, $U L N$ upper limit normal, $H R$ hazard ratio, $C I$ confidence interval, $U L N$ upper limit normal

proportion of patients with PS of 2 has decreased; the international AGC clinical trials-Trastuzumab for Gastric cancer (ToGA) [5] and The Avastin in Gastric Cancer (AVAGAST) [17]—enrolled 10 and 6\%, respectively, of patients with PS of 2. Second, peritoneal metastasis was not a significant prognostic factor in the data set of our study. In the SPIRITS and G-SOX trials, the proportions of patients with peritoneal metastasis were 29 and $19 \%$, respectively, and the proportion of patients with peritoneal metastasis in the cohort analyzed for the RMH index was $16 \%$ of patients. As we previously reported in our paper proposing the JCOG index [14], peritoneal metastasis can be classified into two clinically different types based on tumor burden. While severe peritoneal metastasis, which can be diagnosed by imaging findings such as ascites and a
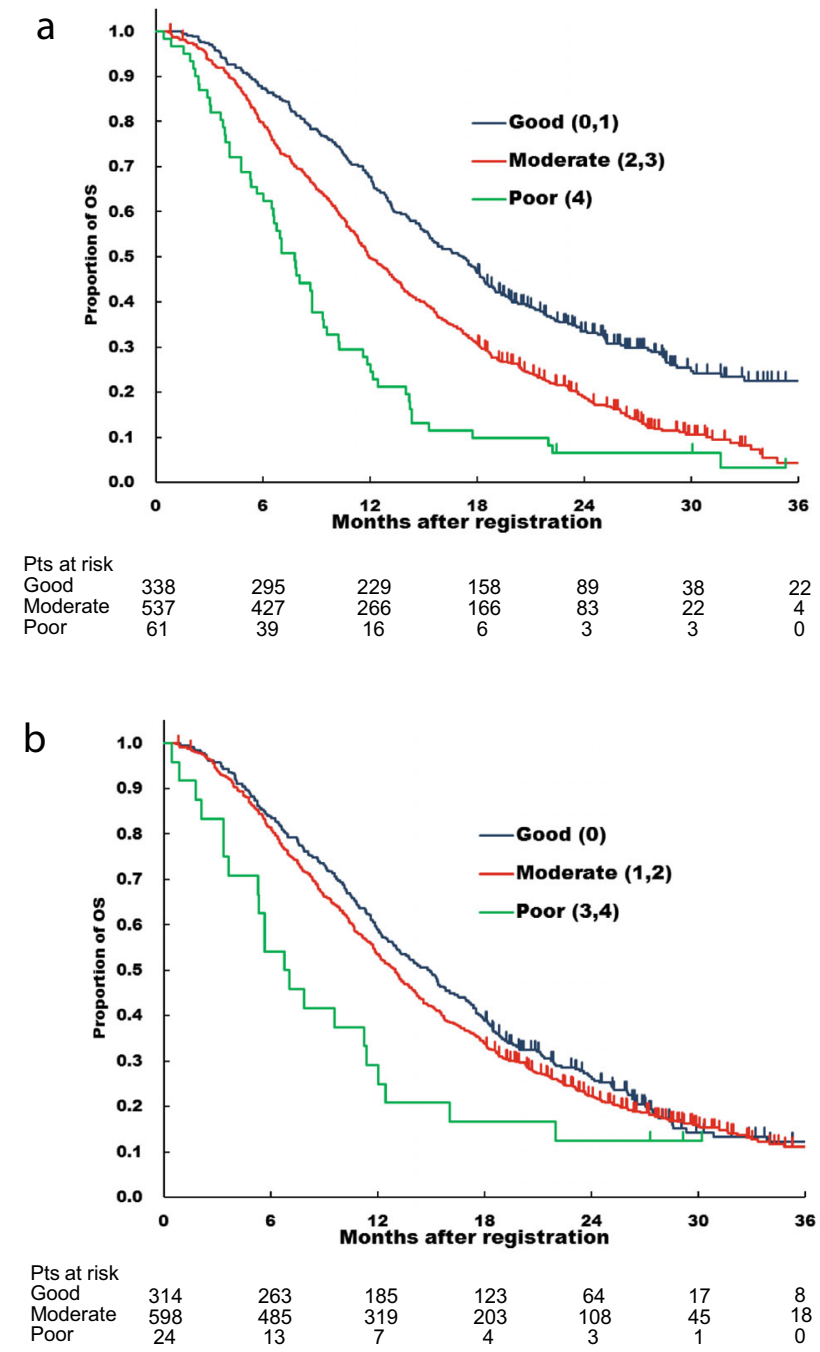

Fig. 3 Applicability of Japan Clinical Oncology Group (JCOG) and Royal Marsden Hospital (RMH) Prognostic indices to combined analysis. a Survival curves of the three groups in this study classified according to the JCOG prognostic index. Good $(0,1), 0$ or 1 risk factor; moderate $(2,3), 2$ or 3 risk factors; poor (4), 4 risk factors. b Survival curves of the three groups in this study classified according to the RMH prognostic index. Good (0), no risk factors; moderate (1, 2), 1 or 2 risk factors; poor $(3,4), 3$ or 4 risk factors

peritoneal nodules, leads to deterioration in the patient's quality of life and is associated with poor survival, minute peritoneal metastases that are undetectable by imaging and can be diagnosed at laparotomy only have a smaller impact on survival. Because the SPIRITS and G-SOX trials excluded patients with severe peritoneal metastases, the proportion of patients with minute peritoneal metastases should be relatively higher; the impact of peritoneal metastasis, which was a factor in the RMH index, was small in this study. Third, many fewer patients have undergone prior gastrectomy in Western countries than in Japan. Although the data used for the RHM index did not show the proportion of patients undergoing prior 
Table 3 Applicability of JCOG prognostic index to the combined analysis (A) JCOG index and (B) RMH index

\begin{tabular}{lccll}
\hline Risk group (no. of risk factors) & $N(\%)$ & MST (months) $(95 \%$ CI) & HR (95\% CI) & $P$ value \\
\hline A) & & & & \\
$\quad$ Good $(0,1)$ & $338(36)$ & $17.2(15.2-18.4)$ & 1 & - \\
$\quad$ Moderate $(2,3)$ & $537(57)$ & $12.0(11.3-13.2)$ & $1.71(1.46-2.01)$ & $<0.0001$ \\
Poor $(4)$ & $61(7)$ & $7.8(6.0-8.8)$ & $3.32(2.47-4.46)$ & $<0.0001$ \\
(B) & & & & \\
Good $(0)$ & $314(34)$ & $14.9(12.9-16.3)$ & 1 & - \\
Moderate $(1,2)$ & $598(64)$ & $13.0(11.9-13.9)$ & $1.10(0.94-1.28)$ & 0.23 \\
Poor $(3,4)$ & $24(3)$ & $6.9(3.6-11.4)$ & $1.98(1.26-3.10)$ & 0.003 \\
\hline
\end{tabular}

$N$ numbers, $M S T$ median survival time, $C I$ confidence interval, $H R$ hazard ratio gastrectomy, they mentioned in their validation study, "compared with those in the Asian population, far fewer patients in our study population would have undergone prior resection [18]." Generally, recurrent patients have a smaller tumor burden than unresectable patients, because follow-up examinations after curative surgery can detect asymptomatic recurrent disease. Prior gastrectomy can be a prognostic factor in a cohort that includes many recurrent patients. Taken together, the JCOG index seems to be suitable for use in clinical trials, while it could be speculated that the $\mathrm{RMH}$ index might be more suitable for clinical practice containing more patients in poor condition. It will be compelling in the future to see whether the JCOG index can surpass the RMH index for evaluation of the non-trial based (or the real world) population.

The Global Advanced/Adjuvant Stomach Tumor Research through International Collaboration (GASTRIC) project performed a meta-analysis of 21 RCTs (3619 patients), which included both Eastern and Western populations. It reported that PS $\geq 1$, disease status (metastatic versus locally advanced), number of metastatic organs, location of metastasis (such as peritoneal metastasis), and prior surgery were significant prognostic factors for AGC patients treated with systemic chemotherapy. Interestingly, geographic area was not a prognostic factor (Asia and the USA with Europe as reference) [19]. Because locally advanced disease can be scored 0 for the number of metastatic organs, there was high concordance between our present study and the GASTRIC project for PS, number of metastatic sites, and prior surgery (laboratory data, including ALP, were not assessed in the GASTRIC project). It has long been conceived that ALP represents not only liver function but also tumor volume in the liver and bone metastasis, even for patients with a single or few organ metastases. Thus, we think that the JCOG prognostic index consists of three components, as follows: the patient's condition (such as PS); tumor volume, as represented by the ALP level and prior gastrectomy; and tumor aggressiveness (such as the number of metastatic organs).
Several recent studies have reported that genetic alterations are prognostic factors in advanced gastric cancer. Fuse et al. reported on the impact of HER2, EGFR, and c-MET status on OS in AGC patients. They suggested that c-MET-positive status was associated with poor prognosis, whereas HER2 and EGFR status did not significantly affect OS without anti-HER2 and anti-EGFR therapy [20]. However, because trastuzumab has a survival benefit for HER2-positive gastric cancer patients, HER2 status can be a marker of favorable survival also for advanced gastric cancer like breast cancer. The combination of our prognostic index with molecular information would enable us to create a more accurate prognostic index in the near future.

Furthermore, Grenader et al. reported a new candidate for a prognostic factor in their post hoc study of the phase III Randomized ECF for Advanced and Locally Advanced Esophagogastric Cancer 2 (REAL-2) trial [3], an elevated neutrophil-to-lymphocyte ratio (NLR), which had a significantly negative prognostic effect [21]. The NLR may represent the immune status of gastric cancer patients. Immune status may be an important prognostic marker, especially when an immune check point inhibitor is introduced to the treatment of gastric cancer. Unfortunately, since we did not have data on NLR, its clinical significance could not be validated in our study.

This study has limitations. First, we could not investigate the impact of HER2 status, because the patients enrolled in the SPIRITS and G-SOX trials were not tested for HER2 expression. Second, the main limitation is that the SPIRITS and G-SOX trials that were used for validation were conducted only in Japan and were not global studies. Some geographical differences in the types of treatments for AGC may be a concern. However, patients participating in clinical trials tend to be similar nowadays because of selection by similar eligibility criteria. First-line chemotherapy is also substantially the same: fluoropyrimidines and platinum are the most favored worldwide. Furthermore, as we already mentioned, geographic area was not a prognostic factor in the GASTRIC project. For the 
next step, we are planning to validate our index in multinational studies or HER2-positive populations.

\section{Conclusions}

The JCOG prognostic index was validated by data from Japanese patients with AGC enrolled in the randomized phase III SPIRITS and G-SOX trials. Three risk groups stratified by the JCOG prognostic index showed highly significant survival differences. Our prognostic index might provide increased accuracy for patient stratification in future clinical trials. Further studies are needed to apply this index to the Western population.

\section{Compliance with ethical standards}

\section{Funding None.}

Conflict of interest Daisuke Takahari has received honoraria from Taiho Pharmaceutical, Yakult Honsha, and Eli Lilly Japan K.K. Narikazu Boku has received honoraria from Taiho Pharmaceutical and Yakult Honsha. Ichinosuke Hyodo has received honoraria from Taiho Pharmaceutical, Yakult Honsha, Chugai, and Daiichi-Sankyo Pharmaceutical Companies. Junki Mizusawa and Wasaburo Koizumi have nothing to disclose.

Human rights statement and informed consent All procedures were in accordance with the ethical standards of the responsible committee on human experimentation (institutional and national) and with the Helsinki Declaration of 1964 and later versions. This analysis was approved by the multicenter Research and Ethics Committees at the Cancer Institute Hospital.

\section{References}

1. Torre LA, Bray F, Siegel RL, Ferlay J, Lortet-tieulent J, Jemal A. Global cancer statistics, 2012. CA a cancer. J Clin. 2015;65(2):87-108.

2. http://ganjoho.jp/reg_stat/sitemap.html. Accessed 29 Aug 2016.

3. Cunningham D, Starling N, Rao S, Iveson T, Nicolson M, Coxon $\mathrm{F}$, et al. Capecitabine and oxaliplatin for advanced esophagogastric cancer. N Engl J Med. 2008;358(1):36-46.

4. Van Cutsem E, Moiseyenko VM, Tjulandin S, Majlis A, Constenla M, Boni C, et al. Phase III study of docetaxel and cisplatin plus fluorouracil compared with cisplatin and fluorouracil as firstline therapy for advanced gastric cancer: a report of the V325 Study Group. J Clin Oncol. 2006;24(31):4991-7.

5. Bang Y-J, Van Cutsem E, Feyereislova A, Chung HC, Shen L, Sawaki A, et al. Trastuzumab in combination with chemotherapy versus chemotherapy alone for treatment of HER2-positive advanced gastric or gastro-oesophageal junction cancer (ToGA): a phase 3, open-label, randomised controlled trial. Lancet. 2010;376(9742):687-97.

6. Solal-Céligny P, Roy P, Colombat P, White J, Armitage JO, Arranz-Saez R, et al. F FLIPI: follicular lymphoma international prognostic index. Blood. 2004;104(5):1258-65.

7. Motzer RJ, Mazumdar M, Bacik J, Berg W, Amsterdam AFJ. Survival and prognostic stratification of 670 patients with advanced renal cell carcinoma. J Clin Oncol. 1999;17(8):2530-40.

8. Köhne CH, Cunningham D, Di Costanzo F, Glimelius B, Blijham $\mathrm{G}$, Aranda E, et al. Clinical determinants of survival in patients with 5-fluorouracil-based treatment for metastatic colorectal cancer: results of a multivariate analysis of 3825 patients. Ann Oncol. 2002;13(2):308-17.

9. Chau I, Norman AR, Cunningham D, Waters JS, Oates J, Ross PJ. Multivariate prognostic factor analysis in locally advanced and metastatic esophago-gastric cancer-pooled analysis from three multicenter, randomized, controlled trials using individual patient data. J Clin Oncol. 2004;22(12):2395-403.

10. Boku N, Yamamoto S, Fukuda H, Shirao K, Doi T, Sawaki A, et al. Fluorouracil versus combination of irinotecan plus cisplatin versus S-1 in metastatic gastric cancer: a randomised phase 3 study. Lancet Oncol. 2009;10(11):1063-9.

11. Lee J, Lim T, Uhm JE, Park KW, Park SH, Lee SC, et al. Prognostic model to predict survival following first-line chemotherapy in patients with metastatic gastric adenocarcinoma. Ann Oncol. 2007;18(5):886-91.

12. Kim JG, Ryoo B-Y, Park YH, Kim B-S, Kim T-Y, Im Y-H, et al. Prognostic factors for survival of patients with advanced gastric cancer treated with cisplatin-based chemotherapy. Cancer Chemother Pharmacol. 2008;61(2):301-7.

13. Koo DH, Ryoo BY, Kim HJ, Ryu MH, Lee SS, Moon JH, et al. A prognostic model in patients who receive chemotherapy for metastatic or recurrent gastric cancer: validation and comparison with previous models. Cancer Chemother Pharmacol. 2011;68(4):913-21.

14. Takahari D, Boku N, Mizusawa J, Takashima A, Yamada Y, Yoshino T, et al. Determination of prognostic factors in Japanese patients with advanced gastric cancer using the data from a randomized controlled trial, Japan Clinical Oncology Group 9912. Oncologist. 2014;19(4):358-66.

15. Koizumi W, Narahara H, Hara T, Takagane A, Akiya T, Takagi M, et al. S-1 plus cisplatin versus S-1 alone for first-line treatment of advanced gastric cancer (SPIRITS trial): a phase III trial. Lancet Oncol. 2008;9(3):215-21.

16. Yamada Y, Higuchi K, Nishikawa K, Gotoh M, Fuse N, Sugimoto N, et al. Phase III study comparing oxaliplatin plus S-1 with cisplatin plus S-1 in chemotherapy-naïve patients with advanced gastric cancer. Ann Oncol. 2015;26(1):141-8.

17. Ohtsu A, Shah MA, Van Cutsem E, Rha SY, Sawaki A, Park SR, et al. Bevacizumab in combination with chemotherapy as firstline therapy in advanced gastric cancer: a randomized, doubleblind, placebo-controlled phase III study. J Clin Oncol. 2011;29(30):3968-76.

18. Chau I, Ashley S, Cunningham D. Validation of the Royal Marsden Hospital prognostic index in advanced esophagogastric cancer using individual patient data from the REAL 2 study. J Clin Oncol. 2009;27(19):3-4.

19. Pozzo C, Ohashi Y. Meta-analysis of randomized trials assessing the influence of chemotherapy and prognostic factor in advanced/ adjuvant gastric cancer. J Clin Oncol. 2009;27(15s) (suppl; abstr 4550).

20. Fuse N, Kuboki Y, Kuwata T, Nishina T, Kadowaki S, Shinozaki E, et al. Prognostic impact of HER2, EGFR, and c-MET status on overall survival of advanced gastric cancer patients. Gastric Cancer. 2016;19(1):183-91.

21. Grenader T, Waddell T, Peckitt C, Oates J, Starling N, Cunningham D, et al. Prognostic value of neutrophil-to-lymphocyte ratio in advanced oesophago-gastric cancer: exploratory analysis of the REAL-2 trial. Ann Oncol. 2016;27(4):687-92. 